\title{
Associations with monetary values do not influence access to awareness for faces
}

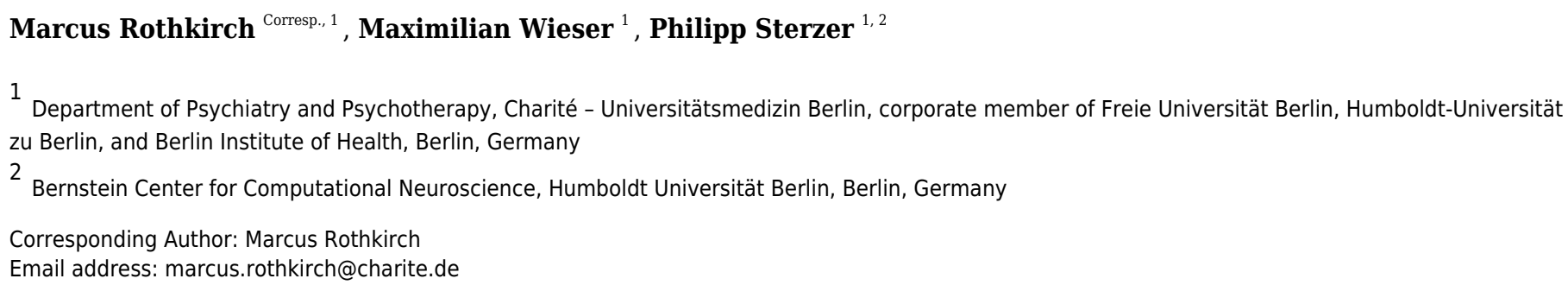

Human faces can convey socially relevant information in various ways. Since the early detection of such information is crucial in social contexts, socially meaningful information might also have privileged access to awareness. This is indeed suggested by previous research using faces with emotional expressions. However, the social relevance of emotional faces is confounded with their physical stimulus characteristics. Here, we sought to overcome this problem by manipulating the relevance of face stimuli through classical conditioning: Participants had to learn the association between different face exemplars and high or low amounts of positive and negative monetary outcomes. Before and after the conditioning procedure, the time these faces needed to enter awareness was probed using continuous flash suppression, a variant of binocular rivalry. While participants successfully learned the association between the face stimuli and the respective monetary outcomes, faces with a high monetary value did not enter visual awareness faster than faces with a low monetary value after conditioning, neither for rewarding nor for aversive outcomes. Our results tentatively suggest that behaviorally relevant faces do not have privileged access to awareness when the assessment of the faces' relevance is dependent on the processing of face identity, as this requires complex stimulus processing that is likely limited at pre-conscious stages. 


\section{Associations with monetary values do not influence 2 access to awareness for faces}

3

4

5

6

7
Marcus Rothkirch $^{1}$, Maximilian Wieser ${ }^{1}$, Philipp Sterzer ${ }^{1,2}$

${ }^{1}$ Department of Psychiatry and Psychotherapy, Charité - Universitätsmedizin Berlin, corporate member of Freie Universität Berlin, Humboldt-Universität zu Berlin, and Berlin Institute of Health, Berlin, Germany

${ }^{2}$ Bernstein Center for Computational Neuroscience, Humboldt-Universität zu Berlin, Berlin, Germany

Corresponding Author:

Marcus Rothkirch

Charitéplatz 1, Berlin, 10117, Germany

Email address: marcus.rothkirch@charite.de

(18

(19)

(1)

2

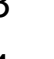

25

27


38

39

40

41

42

43

44

45

46

47

48

49

50

51

52

53

54

55

56

57

58

59

60

61

62

63

64

65

66

67

68

69

70

71

72

73

74

75

76

77

\section{Introduction}

The ability to identify and to rapidly read information from human faces has a pivotal role in social contexts. Since the multitude of information conveyed by faces goes far beyond the image per se, different cognitive systems are involved in face processing. Faces are thus a popular tool to assess what types of information can be processed without the observer's awareness or have preferential access to awareness (Axelrod, Bar \& Rees, 2015; Madipakkam \& Rothkirch, 2019). A particular focus of previous research in this context was on the question whether the social meaning of faces is already processed at pre-conscious stages, thereby facilitating conscious awareness of faces that convey socially relevant information. Indeed, facial cues signaling threat (Yang, Zald \& Blake, 2007; Yang \& Yeh, 2018), trustworthiness (Stewart et al., 2012; Getov et al., 2015), or positive emotions (Stein \& Sterzer, 2012) seem to accelerate the awareness of faces. However, emotional expressions and other social characteristics of faces, such as trustworthiness or dominance, are inextricably linked to physical stimulus properties, like contrast, luminance, or spatial frequencies. In fact, the prioritization of emotional faces can be largely explained by differences in such physical stimulus properties (Stein \& Sterzer, 2012; Gray et al., 2013; Hedger et al., 2016; Stein et al., 2018). To be able to unequivocally attribute differences in the access to awareness of faces to their behavioral relevance, however, the influence of physical stimulus properties should be ruled out first (Moors et al., 2019).

An elegant way to circumvent the inherent confound between physical stimulus properties and higher-level relevance is to ascribe behavioral relevance to faces in a systematic and controlled manner. That way, the association between the physical characteristics and the relevance of stimuli can be balanced out across observers. Anderson et al. (2011) followed such an approach by pairing faces with positive, negative, or neutral gossip. They observed that faces previously paired with negative gossip dominated visual awareness during a following binocular rivalry task. In subsequent studies, in contrast, affective biographical information did not influence observers' awareness of faces, suggesting a rather limited impact of such information on visual awareness (Rabovsky, Stein \& Abdel Rahman, 2016; Stein et al., 2017). One reason for these conflicting findings might be that the relevance of social information depends on each individual's evaluation of this information. Indeed, the time for complex stimuli to reach awareness can depend on the subjectively experienced value of the stimulus (Schmack et al., 2016) or certain personality traits of the observer (Madipakkam et al., 2019).

In the present study, we chose to systematically pair images of faces with high or low amounts of monetary reward and punishment. Manipulating the behavioral relevance of faces by means of monetary incentives has several advantages over using verbal descriptions. In comparison to the latter, monetary values are quantitative, which implies that different conditions can be clearly defined. In this regard, different conditions are set by different amounts of the same unit, whereas for biographical information descriptions of positive behaviors or traits, for example, are 
78

79

80

81

82

83

84

85

86

87

88

89

90

91

92

93

94

95

96

97

98

99

100

101

102

103

104

105

106

107

108

109

110

111

112

113

114

115

116

117

compared to entirely different descriptions that are supposed to be identified as neutral. Thus, monetary values allow for a systematic control of the behavioral relevance of faces and are intersubjectively meaningful. The association of stimuli with monetary incentives by means of classical conditioning can modulate the subjective value of the stimuli to a similar extent as to primary reinforcers (Delgado, Labouliere \& Phelps, 2006; Lehner et al., 2016). Moreover, such associations have the potency to influence visual attention, such that stimuli associated with higher monetary values capture and guide attention more strongly than stimuli associated with lower values (Austin \& Duka, 2010; Bucker \& Theeuwes, 2017, 2018). It is reasonable to assume that such learned associations can also affect how quickly stimuli enter awareness, since stimuli previously paired with high monetary reward are more often consciously perceived during rapid sequences of visual stimuli than stimuli paired with low reward (LeganesFonteneau, Scott \& Duka, 2018). In our study, participants had to learn the association between face stimuli and monetary values. Before and after the learning phase, the same faces were presented under breaking continuous flash suppression (bCFS) to measure the time they require to get access to awareness. BCFS is a variant of binocular rivalry, in which two different stimuli are presented to the two different eyes. Unlike binocular rivalry, however, for bCFS a dynamic stimulus (i.e. the masking stimulus) is presented to one eye, while a static stimulus (i.e. the target) is presented to the other eye. This results in the initial dominance of the masking stimulus, allowing for a greater control of stimulus suppression in comparison to the rather stochastic nature of perceptual states during binocular rivalry. We hypothesized that if learned values facilitate faces' access to awareness, there should be a stronger decrease in response times after the conditioning session for faces associated with a high monetary compared to a low monetary value. The inclusion of monetary reward as well as punishment further enabled us to detect valence-specific effects of learned values on visual awareness.

\section{Materials \& Methods}

\section{Participants}

Twenty-four participants (13 females; age: 18 - 35 years, $M=24.54 \pm 4.36$ standard deviation [SD]) took part in the experiment. Five participants were left-handed, all other participants were right-handed. All participants had normal or corrected-to-normal vision and written informed consent was obtained from each participant prior to their participation in the experiment. The study was approved by the local ethics committee of the Charité - Universitätsmedizin Berlin (EA1/301/13) and performed in accordance with the Declaration of Helsinki. The final sample size was based on a sequential testing approach using Bayes Factors (Schönbrodt et al., 2017). More specifically, we planned to calculate Bayes Factors (BF10) for our main analysis after every new batch of 24 participants, since this sample size is needed to completely counterbalance the association between stimulus exemplars and monetary values across participants. Our aim was to continue data collection until these Bayes Factors would either exceed a threshold of 3 , 
118 which indicates evidence in favour of the alternative hypothesis, or fall below a threshold of $1 / 3$, 119 indicating evidence in favour of the null hypothesis. Since this condition was already met after

120

121

122

123

124

125

126

127

128

129

130

131

132

133

134

135

136

137

138

139

140

141

142

143

144

145

146

147

148

149

150

151

152

153

154

155

156

157 the inclusion of 24 participants, we stopped data collection at this stage.

\section{Stimuli and Apparatus}

The face stimuli used in the study were grey-scale photographs of four different female faces with a neutral expression, taken from the NimStim Set of Facial Expressions (Tottenham et al., 2009; image IDs: 01, 07, 09, 17). All four images were similar in global contrast (root mean square contrast between 0.16 and 0.20 ) and luminance (mean luminance between $27.49 \mathrm{~cd} / \mathrm{m}^{2}$ and $\left.31.35 \mathrm{~cd} / \mathrm{m}^{2}\right)$. All stimuli were presented on a uniformly grey background $\left(30.28 \mathrm{~cd} / \mathrm{m}^{2}\right)$. Participants viewed the screen through a mirror stereoscope providing separate visual input to the two eyes. Each participant's head was stabilized by a chin rest at a viewing distance of 60 $\mathrm{cm}$. All stimuli were presented using MATLAB (The MathWorks, Natick, MA, USA) and Psychtoolbox-3 (http://psychtoolbox.org/) on a 19 inch CRT monitor (resolution: 1024 x 768 Px, refresh rate: $60 \mathrm{~Hz})$.

\section{Procedure}

The experiment consisted of three phases: 1) a pre-conditioning phase to measure baseline response times, 2) a conditioning phase during which different faces were paired with monetary outcomes, and 3) a post-conditioning phase that was identical to the pre-conditioning phase, intended to assess the change in response times after the conditioning had taken place. In the initial pre-conditioning phase (Figure 1A), different face exemplars were presented under continuous flash suppression. At the beginning of each trial, a black rectangle $\left(10^{\circ} \times 10^{\circ}\right)$ and a black fixation cross in its center $\left(0.68^{\circ} \times 0.68^{\circ}\right)$ were presented to each eye. The rectangle and the cross were visible throughout the whole experimental phase. After a fixation duration of $2 \mathrm{~s}$, high-contrast dynamic mask stimuli consisting of circles and squares of various colors and sizes were flashed to a randomly selected eye at a frequency of $10 \mathrm{~Hz}$. Simultaneously, a face image $\left(3.75^{\circ} \times 3.75^{\circ}\right)$ that was located within one of the four quadrants of the black rectangle was presented to the other eye. The contrast of the face stimulus linearly increased from $0 \%$ to $100 \%$ during the initial $2 \mathrm{~s}$. After that, the face remained at full contrast until the end of the trial. A trial ended when the participant gave a manual response or, if no response was made, after $15 \mathrm{~s}$. Participants' task was to indicate the location of the face, that is, the quadrant in which the face appeared, as fast and as accurately as possible by pressing one of four designated keys on the keyboard. This part of the experiment comprised 96 trials. The combination of the face exemplar, the location of the face, and the eye to which the face was presented was counterbalanced and randomized across trials.

In the second phase, participants had to learn the association between the face exemplars and monetary outcomes by means of classical conditioning (Figure 1B). In each trial, one of the faces that were already presented in the first part of the experiment was shown in the center of the screen. Below the face, a positive or negative monetary value was displayed. The face and the 
158

159

160

161

162

163

164

165

166

167

168

169

170

171

172

173

174

175

176

177

178

179

180

181

182

183

184

185

186

187

188

189

190

191

192

193

194

195

196

197

value were presented for $5 \mathrm{~s}$. After the offset of the face and the associated monetary value, a blank screen was presented for a randomized interval between $1 \mathrm{~s}$ and $1.6 \mathrm{~s}$ before the next trial started. Each face was associated with one of four different monetary values: $-2 €,-0.1 €,+0.1 €$, and $+2 €$. In $75 \%$ of the trials, the face was depicted with its associated monetary value. In the remaining trials, an outcome of $0 €$ was presented along with the face. Such a probabilistic reinforcement schedule was chosen to maintain participants' attention to the stimuli and the task, as for classical conditioning with monetary outcomes attention is preferably directed towards partially predictive stimuli in comparison to fully predictive stimuli (Austin \& Duka, 2010). Participants were instructed to passively view the stimuli and to memorize the association between the faces and the monetary values as well as possible. They were further informed that the monetary value depicted in a trial would be counted towards their overall payoff. Thus, for a trial, in which a face was presented together with ' $+2 €$ ', for instance, $2 €$ would be added to their payoff, while for trials with ' $-2 €$ ' $2 €$ would be subtracted from their payoff. Since unbeknown to participants each monetary value was presented equally often, the outcome for this part of the conditioning phase amounted to zero. Thus, participants' outcome was solely defined by their accuracy in the query trials (see below). This phase of the experiment comprised four blocks, during which each face was presented four times. The order of the face exemplars was randomized and the association between the face exemplars and the monetary values was fully counterbalanced across participants. The association was further kept constant across all four blocks.

To assess whether participants were indeed able to learn these associations, query trials were added at the end of each block. Each face was presented once during these query trials. In contrast to the conditioning trials, a question mark and all four different monetary values were displayed below the face in random order. Participants were required to select the value that was associated with the respective face by pressing one of four designated buttons. The face and the monetary values were presented until a response was made. Participants were informed that monetary reimbursement for their participation in the experiment would depend on the accuracy of their choices during these query trials. After the experiment, participants indeed received the amount of money that they accumulated during the conditioning phase. More specifically, a correct assignment of a monetary reward to the respective face would yield an addition of $+2 €$ or $+0.1 €$, respectively, to their payoff. A wrong response to faces associated with reward did not result in a monetary gain. For faces associated with a monetary punishment, participants had to assign the correct monetary value to avoid a monetary loss. This means that a correct assignment of $-2 €$ or $-0.1 €$ to the respective face did not yield a monetary gain or loss. However, if a participant assigned a wrong value to a punishment-related face, this resulted in a loss of $-2 €$ or $0.1 €$, respectively. Thus, participants expected that their payoff was dependent on both, the passively viewed pairings of monetary values and faces as well as their performance during the query trials. This procedure served two purposes: 1) to increase the relevance of the high-valued compared to the low-valued faces, and 2) to increase participants' attention to all face stimuli during the query trials so that the accuracies in the query trials would provide an optimal account 
198

199

200

201

202

203

204

205

206

207

208

209

210

211

212

213

214

215

216

217

218

219

220

221

222

223

224

225

226

227

228

229

230

231

232

233

234

235

of participants' learning progress. Note that in case of negative values participants had to respond accurately to avoid monetary losses during the query trials.

The post-conditioning phase, which followed directly after the conditioning phase, was fully identical to the pre-conditioning phase. After the experiment, participants rated how much they felt motivated by the different monetary values to memorize the faces on a visual analog scale ranging from 0 to 5 .

\section{Data Analysis}

Participants' learning performance during the conditioning phase was assessed on the basis of their responses in the query trials. For each of the four blocks, each participant's accuracy in assigning the monetary value to each face exemplar was computed. The chance level for each block was .25. To evaluate participants' sensitivity for reward and punishment, we computed each participant's response bias during the query trials. For reward-related faces the response bias is computed on the basis of the $z$-transformed hits $\left(H_{R}\right)$ and false alarm rates $\left(F A_{R}\right)$ as follows:

$c_{R}=-\frac{z\left(H_{R}\right)+z\left(F A_{R}\right)}{2}$

However, since participants have to assign a positive or negative value to each face, the response biases for reward and punishment are not independent of each other. More specifically, an increase in the false alarm rate for reward leads to a decrease in the hit rate for punishment $\left(\mathrm{H}_{\mathrm{P}}\right)$ by the same amount, such that: $\mathrm{z}\left(\mathrm{FA}_{\mathrm{R}}\right)=-\mathrm{z}\left(\mathrm{H}_{\mathrm{P}}\right)$. Thus, the formula above can be rewritten as: $c_{R}=-\frac{z\left(H_{R}\right)-z\left(H_{P}\right)}{2}$

Since a bias towards reward $\left(c_{R}\right)$ automatically implies a bias away from punishment $\left(c_{P}\right)$ such that $c_{R}=-c_{P}$, we only report the reward response bias. For $c_{R}$, a positive bias denotes a stronger sensitivity for reward, while a negative bias denotes a stronger sensitivity for punishment. For the bCFS phase before and after the conditioning phase, trials in which participants responded incorrectly (percentage of trials: $\mathrm{M}=3.19 \%, \mathrm{SD}=2.75 \%$ ) or failed to give a response until the end of a trial $(\mathrm{M}=0.61 \%, \mathrm{SD}=1.26 \%)$ were discarded from further analysis.

Furthermore, response times below $200 \mathrm{~ms}$ were considered anticipatory responses and were also discarded from further analysis (percentage of trials: $\mathrm{M}=0.61 \%, \mathrm{SD}=1.26 \%$ ). To compare the response times between the different conditions, we followed two approaches. For the first approach, we computed the median response time for each participant and condition before and after conditioning. We used the median instead of the mean to account for the skewness of response time distributions, which is line with other bCFS studies (e.g. Gayet et al., 2016; Gayet \& Stein, 2017; Han, Blake \& Alais, 2018). We then subtracted the median response times of the pre-conditioning phase from the median response times of the post-conditioning phase, which resulted in a measure for the change of response times for each condition and participant. Finally, we performed two paired t-tests to compare the mean change in response times between high and low reward as well as between high and low punishment. The alpha level of these two 
236 t-tests was adjusted to .025 to account for multiple comparisons. For the second approach, we 237 analyzed the response time distributions in more depth by computing hierarchical shift functions 238 (Rousselet \& Wilcox, 2019), which can be more sensitive to response time differences, 239 especially when they are restricted to early or late responses. To this end, we computed the 240 deciles of the response time distribution for each condition and participant before and after 241 conditioning. In a next step, we subtracted the deciles of the pre-conditioning phase from the 242 deciles of the post-conditioning phase. The resulting values thus indicate the change in response 243 times for each segment of the whole distribution, where lower deciles reflect faster responses and higher deciles slower responses. For each decile, we then performed a paired t-test to compare the changes in response times of the high reward to the low reward condition and of the high punishment to the low punishment condition. As this amounts to 18 different t-tests in total, we adjusted the alpha level of the t-tests to .003 . Thus, we adjusted the significance level for the number of tests across reward and punishment, because our aim was to control the maximum experiment-wise error rate (Bender \& Lange, 2001). Our reasoning for applying such a rigorous adjustment of the alpha level was that the underlying hypothesis of an influence of learned values on bCFS response times would be supported by a difference in response times for either reward or punishment.

253 To probe potential time-dependent effects of learned values, we additionally split the post254 conditioning phase into two halves. For statistical inference, we performed repeated-measures ANOVAs with the factors time (first half vs. second half of the post-conditioning phase) and value (high value vs. low value). Separate ANOVAs were performed for reward-related and punishment-related stimuli. The focus of this analysis was on the interaction between time and value, since a statistically significant interaction would signify an influence of the monetary value that was dependent on time. Note that this analysis was performed on the median response time differences between the pre- and post-conditioning phase.

261

262 Finally, we performed two generalized linear mixed-effects models on the single trial data, one for reward-related and one for punishment-related stimuli. This analysis was intended to take into account the variability of the effect of interest in dependence on the repetition of the stimuli and the association between the different face exemplars and the monetary values. We defined a Gamma distribution as the distribution of our response variable, since it provides a plausible approximation to the processes reflected in response times (Lo \& Andrews, 2015). We included the interaction of face value, bCFS phase, face exemplar, and trial number as well as all other interactions and main effects of these variables as fixed effects in our models, for which we used effects coding. For the random effects term, we defined the theoretically maximal model by including subject as a random factor together with the by-subject random intercept and the bysubject random slopes of all fixed factors (Barr et al., 2013). Both models thus had the following 272 structure:

273 RT value $*$ phase $*$ exemplar $*$ trial $+(1+$ value $*$ phase $*$ exemplar $*$ trial $\mid$ subject $)$

274 The estimate of each fixed-effects predictor was then tested against zero by means of individual 275 t-tests. 
276

277

278

279

280

281

282

283

284

285

286

287

288

289

290

291

292

293

294

295

296

297

298

299

300

301

302

303

304

305

306

307

308

309

310

311

312

313

314

In addition to frequentist inference statistics, we computed Bayes Factors (BF10) for each t-test, using a default Cauchy prior of 0.707. For ANOVAs we computed BF10 directly from the Fvalues of the ANOVA statistics (Faulkenberry, 2018). In line with previous suggestions (Wetzels \& Wagenmakers, 2012), we interpret BF10 $>3$ as evidence for the alternative hypothesis and $\mathrm{BF} 10<1 / 3$ as evidence for the null hypothesis. We also report BF01 to clarify which analyses yielded evidence for the null hypothesis. In this case, BF01 $>3$ indicates evidence for the null hypothesis.

\section{Results}

\section{Classical conditioning}

At the end of every block in the conditioning phase, participants had to indicate the associated monetary value for each presented face. Figure 2A depicts the accuracy of these responses for the four different blocks. Since participants had to make four choices in each block, the chance level corresponds to an accuracy of .25 for each block. Binomial tests indicated that the response accuracies across all four blocks exceeded chance level for all participants $(\mathrm{p} \leq .002, \mathrm{BF} 10 \geq$ 10.10). Figure $2 \mathrm{~B}$ shows the response accuracies across all four blocks separately for each value condition. As for each value condition the majority of participants did not commit any mistake during the query trials, the median accuracy for each condition amounted to 1 . Thus, participants quickly and successfully learned the associations between the face exemplars and the monetary outcomes for each of the different monetary values. After the experiment, participants rated their motivation for each monetary value on a visual analog scale ranging from 0 to 5 . In comparison to a low reward of $0.1 €(\mathrm{M}=1.51 \pm 0.28$ standard error of the mean [SEM]), participants felt substantially more motivated by the high reward of $2 €(\mathrm{M}=2.98 \pm 0.36$ SEM; paired t-test: $\mathrm{t}(23)=4.99, \mathrm{p}<.001, \mathrm{BF} 10=521.53)$. The difference in motivation between a low punishment of $0.1 €(M=1.46 \pm 0.30 \mathrm{SEM})$ and a high punishment of $2 €(\mathrm{M}=2.08 \pm 0.37 \mathrm{SEM})$ was less pronounced compared to reward $(\mathrm{t}(23)=2.42, \mathrm{p}=.024, \mathrm{BF} 10=2.35)$, but still statistically significant at a corrected threshold of $\alpha=.025$. The majority of participants $(n=18)$ did not exhibit a response bias to either reward or punishment (i.e. $\left.c_{R}=0\right)$. One participant showed a response bias towards monetary punishment $\left(c_{R}=-0.18\right)$ and five participants showed a response bias towards monetary reward $\left(\mathrm{c}_{\mathrm{R}}=0.24-0.97\right)$.

\section{Response times during breaking-CFS}

Figure 3A shows the change in response times from the pre-conditioning to the post-conditioning phase for the two different reward conditions. For the high reward condition, response times decreased by $501.01 \mathrm{~ms}$ ( $\pm 102.27 \mathrm{~ms}$ SEM), on average, while for the low reward condition we observed an average decrease in response times of $412.74 \mathrm{~ms}( \pm 84.25 \mathrm{~ms} \mathrm{SEM})$. The difference 
315 between the two conditions was not statistically significant $(\mathrm{t}(23)=0.80, \mathrm{p}=.43)$ and the Bayes 316 factor indicated the absence of an effect $(\mathrm{BF} 10=0.29, \mathrm{BF} 01=3.48)$.

317 The average change in response times for the high and low punishment condition are depicted in 318 Figure 3B. Numerically, there was a stronger decrease in response times for the low punishment 319 condition $(\mathrm{M}=-477.85 \pm 97.54 \mathrm{~ms} \mathrm{SEM})$ in comparison to the high punishment condition $(\mathrm{M}=$

320

321

322

323

324

325

326

327

328

329

330

331

332

333

334

335

336

337

338

339

340

341

342

343

344

345

346

347

348

349

350

351

352

353

354 $-401.97 \pm 82.05 \mathrm{~ms}$ SEM). However, the difference between the two conditions was again not statistically significant and indicative of an absence of an effect $(\mathrm{t}(23)=0.65, \mathrm{p}=.52, \mathrm{BF} 10=$ $0.26, \mathrm{BF} 01=3.84$ ).

To rule out baseline differences between conditions that might have masked potential effects of learned values in the post-conditioning phase, we additionally computed response times during the pre-conditioning phase only. Response times for faces that were later paired with a high reward ( $\mathrm{M}=2275.90 \pm 464.57 \mathrm{~ms} \mathrm{SEM})$ did not significantly differ from response times for faces that were later paired with a low reward $(\mathrm{M}=2254.57 \pm 460.21 \mathrm{~ms} \mathrm{SEM} ; \mathrm{t}(23)=0.17, \mathrm{p}=$ $.87, \mathrm{BF} 10=0.22, \mathrm{BF} 01=4.60)$. Similarly, the time to respond to faces later associated with high punishment $(\mathrm{M}=2224.52 \pm 454.08 \mathrm{~ms}$ SEM$)$ was not significantly different from faces later associated with low punishment $(\mathrm{M}=2315.77 \pm 472.71 \mathrm{~ms} \mathrm{SEM} ; \mathrm{t}(23)=0.85, \mathrm{p}=.40, \mathrm{BF} 10=$ $0.30, \mathrm{BF} 01=3.36$ ).

Differences between high and low values, however, might be limited to a specific range of the whole response time distributions, that is, the learned values could specifically affect fast or slow responses, which would not necessarily be captured by measures of central tendencies. We therefore explored the response time distributions in more depth by computing the change between the pre- and the post-conditioning phase for each decile of the whole response time distribution for each condition. For reward-related stimuli (Figure 4A) as well as well as for punishment-related stimuli (Figure 4B), the decrease in response times was more pronounced for slower responses. However, there was no significant difference between high and low values for any of the deciles ( $\mathrm{p} \geq .12$ and $\mathrm{BF} 10 \leq 0.65$ for reward, $\mathrm{p} \geq .12$ and $\mathrm{BF} 10 \leq 1.66$ for punishment). Of note, the differences between high and low reward and punishment, respectively, were close to an uncorrected significance level of .05 for the last deciles. The response time decreases in this decile, however, were numerically stronger for low values compared to high values and as such contrary to our a priori hypothesis.

Due to extinction, potential influences of the monetary associations on response times could have quickly decayed during the post-conditioning phase. Thus, averaging across all responses in the post-conditioning phase might have masked the effects of learned values. To identify potential time-dependent effects, we split the post-conditioning phase into two halves. We performed two repeated-measures ANOVAs with the factors time (first half vs. second half of the postconditioning phase) and value (low value vs. high value). If the influence of learned values was indeed dependent on time such that it quickly faded after the conditioning block, this should be reflected in the interaction between time and value. However, neither for faces previously 
355

356

357

358

359

360

361

362

363

364

365

366

367

368

369

370

371

372

373

374

375

376

377

378

379

380

381

382

383

384

385

386

387

388

389

390

391

392

393

394

associated with reward $(\mathrm{F}(1,23)=0.60, \mathrm{p}=.44, \mathrm{BF} 10=0.28, \mathrm{BF} 01=3.59)$ nor for faces associated with punishment $(\mathrm{F}(1,23)=0.76, \mathrm{p}=.39, \mathrm{BF} 10=0.30, \mathrm{BF} 01=3.31)$ we observed an interaction between time and value.

In line with our main analysis, the parameter estimates for the interaction of the face value and the bCFS phase in the generalized linear mixed-effects models were not statistically significant from zero, neither for reward $\left(\beta=-8.49^{*} 10^{-6}, \mathrm{t}(2194)=-1.87^{*} 10^{-5}, \mathrm{p}>.99\right)$ nor for punishment $\left(\beta=-4.26^{*} 10^{-5}, \mathrm{t}(2193)=-8.62 * 10^{-5}, \mathrm{p}>.99\right)$. There were also no other statistically significant interactions with trial number or face exemplar (all $p>.99$ ).

\section{Exploratory analyses}

While our a priori hypotheses focused on the comparison of the change in response times for high vs. low monetary values, we performed two additional exploratory analyses to examine whether the learned values might have influenced the response times during the bCFS phase in other ways.

For the first analysis, we collapsed our data across high and low values and compared response time changes for all reward-related faces to all punishment-related faces. For reward-related faces, response times decreased by $428.13 \mathrm{~ms}$ ( $\pm 87.39 \mathrm{SEM})$, while for punishment-related faces response times decreased by $443.98 \mathrm{~ms} \mathrm{(} \pm 90.63 \mathrm{SEM})$, on average. The difference between reward and punishment was not statistically significant $(\mathrm{t}(23)=0.19, \mathrm{p}=.85, \mathrm{BF} 10=$ $0.22, \mathrm{BF} 01=4.59$ ). Even when only faces associated with a high value (i.e. high reward vs. high punishment) were considered, the difference between reward and punishment did not reach statistical significance $(\mathrm{t}(23)=1.15, \mathrm{p}=.26, \mathrm{BF} 10=0.39, \mathrm{BF} 01=2.58)$.

In a second exploratory analysis we examined whether the influence of learned values on response times might have been dependent on the learning performance during the conditioning phase. To this end, we computed each participant's accuracy in the query trials across all four conditioning blocks. We then correlated these accuracies against the response time differences (i.e. post- vs. pre-conditioning) during the bCFS blocks, separately for reward- and punishmentrelated faces. The bCFS response time differences were computed by subtracting the change in response times for the low value condition from the change in response times for the high value condition as follows: ([RT post $\left.\left.-\mathrm{RT}_{\text {pre }}\right]_{\text {high reward }}-\left[\mathrm{RT}_{\text {post }}-\mathrm{RT} \mathrm{T}_{\text {pre }}\right]_{\text {low reward }}\right)$ and $\left(\left[\mathrm{RT}_{\text {post }}-\mathrm{RT}_{\text {pre }}\right]_{\text {high }}\right.$ punishment $\left.-\left[\mathrm{RT}_{\text {post }}-\mathrm{RT}_{\text {pre }}\right]_{\text {low punishment }}\right)$. As such, negative values indicate a change in response times in line with our a priori hypotheses, that is, a stronger decrease in response times for the high compared to the low value condition. Since only few participants exhibited low accuracies during the query trials, the distribution of participants' accuracies was heavily skewed. To account for this, we computed Spearman's $\rho$. Neither for reward $(\rho=.30, p=.16, B F 10=0.43$, BF01 $=2.35)$ nor for punishment $(\rho=.13, p=.56, \mathrm{BF} 10=0.19$, BF01 $=5.39)$, accuracies were related to response time differences between conditions. Note that if higher accuracies in the query trials were related to a greater influence of high monetary values on response times, this would be indicated by a negative correlation. Due to the high accuracy during the query trials in the majority of participants, this analysis should be interpreted with caution. 


\section{Discussion}

398

399

400

401

402

403

404

405

406

407

408

409

410

411

412

413

414

415

416

417

418

419

420

421

422

423

424

425

426

427

428

429

430

431

432

433

434

We studied whether learned values, established by means of classical conditioning with monetary reward and punishment, influence access to awareness for faces. While participants successfully learned the association between the different face exemplars and the monetary values, the learned association did not have an influence on their response times. Response times generally decreased from the pre- to the post-conditioning phase. However, this decrease was equally strong for high compared to low reward and for high compared to low punishment. A more in-depth exploration of the response time distributions did not reveal an advantage for faces paired with a higher compared to a lower monetary value either.

Faces can express and convey their relevance in various ways, for instance through their emotional expression or particular facial features such as eye gaze. In the current study, we paired faces with monetary incentives to render them behaviorally relevant. This way, we intended to circumvent a confound between physical stimulus characteristics and higher-level social relevance, which is especially prevalent in the investigation of the awareness of emotional faces (Hedger et al., 2016). While it has previously been suggested that learned associations between affective information and faces affect the faces' potency to dominate awareness (Anderson et al., 2011), subsequent studies did not observe a privileged access to awareness for faces paired with affective information (Rabovsky, Stein \& Abdel Rahman, 2016; Stein et al., 2017). Our findings are in line with the latter studies, while at the same time complementing these previous results by showing that not only biographical information but also monetary incentives fail to facilitate awareness of faces. The approach of associating face stimuli with affective information, however, differs in two aspects from the investigation of the influence of emotional expressions. First, emotional expressions are inherent to a face, while the association with affective information has to be learned. Secondly, the identification of emotional expressions often only requires the processing of certain facial features. A rapid detection of fearful faces, for instance, is likely due to the greater exposure to the iris and the sclera in fearful faces (Whalen et al., 2004). Effects of learned associations, in contrast, likely requires the identification of the faces' whole identity. Since conditioned responses to fear-conditioned faces transfer to novel images of the same face identity (Rehbein et al., 2018), learned associations are indeed, at least partly, related to the face's identity. Thus, the influence of learned affective information, as in our case by means of monetary values, is dependent on a more complex analysis of the stimulus at pre-conscious stages. As the processing of face identity is rather limited under visual masking (Moradi, Koch \& Shimojo, 2005; Amihai, Deouell \& Bentin, 2011), the scope of pre-conscious face processing might not be sufficient to boost faces into awareness that have been coupled with positive or negative outcomes. Similar limitations likely apply to other types of complex stimuli when different exemplars of the same category are paired

PeerJ reviewing PDF | (2020:06:49896:3:0:NEW 7 Jan 2021) 
435 with different outcomes. Though to what extent pre-conscious processing is limited for different 436 stimulus categories has to be further investigated in future studies.

437

438

439

440

441

442

443

444

445

446

447

448

449

450

451

452

453

454

455

456

457

458

459

460

461

462

463

464

465

466

467

468

469

470

471

472

473

474
Participants were clearly able to learn the association between the face exemplars and the respective monetary values. The absence of an influence of the associated values on the access of the faces to awareness can thus not be attributed to a failure or difficulties of participants to learn these associations. There is ample evidence for sustained neural (Rothkirch et al., 2012) and behavioral effects (Raymond \& O'Brien, 2009; Rutherford, O'Brien \& Raymond, 2010; Rothkirch et al., 2013) of previously learned associations between faces and monetary values, indicating that pairing face stimuli with monetary outcomes has the potency to render face stimuli behaviorally relevant for an extended period of time. It must be noted, however, that such associations have been mostly induced by means of instrumental conditioning so far. While classical conditioning with monetary incentives can generally bring about similar effects compared to instrumental conditioning (Delgado, Labouliere \& Phelps, 2006; Bucker \& Theeuwes, 2016, 2017), the specific combination of face stimuli and monetary outcomes in the context of classical conditioning has only rarely been studied so far. However, Trilla Gros, Panasiti \& Chakrabarti (2015) found that EEG responses to faces are influenced by their previous associations with monetary reward and punishment established through classical conditioning. While this shows that classical conditioning with monetary values has the potency to alter neural signals in response to face stimuli, it leaves open the question how such altered neural signals translate into behavioral effects. It has further been demonstrated previously that simple visual stimuli, like gratings, can gain faster access to awareness by means of classical fear conditioning (Gayet et al., 2016), showing that a conditioning approach can, in principle, confer behavioral relevance to visual stimuli such that they access awareness more rapidly. There is the residual possibility that the association between the face stimuli and the monetary values in our study did not effectively change the affective content of the faces, especially because associating faces with monetary incentives by means of classical conditioning might be less effective compared to instrumental conditioning. For fear-conditioning, the effectiveness of the conditioning procedure is usually assessed on the basis of physiological measures, like skin conductance responses. For appetitive conditioning and conditioning with monetary outcomes, in contrast, such a standard physiological measure has not yet been established. Since pupil size promises to be a fruitful measure of the effectiveness of appetitive conditioning (Pietrock et al., 2019), it could be assessed in future studies focusing on the influence of learned stimulus values on the access to awareness. The availability of such a measure would also allow to relate the strength of conditioning in each individual to the effect of learned values on visual awareness (Madipakkam et al., 2016; Vieira et al., 2017). Alternatively or complementary to physiological responses, the effectiveness of the conditioning procedure could further be evaluated on the basis of a control task with clearly visible stimuli. Such a control task, though, would either require a separate sample of participants or would have to be included after the conditioning phase in addition to the bCFS task, in which case the extinction of the conditioned values plays an

Peerj reviewing PDF | (2020:06:49896:3:0:NEW 7 Jan 2021) 
475 important role. Physiological measures, in contrast, could be more easily integrated in the 476 existing experimental design.

477 A further relevant aspect are potential asymmetries in participants' sensitivity to reward or 478 punishment. We assessed participants' sensitivity in our study on the basis of their responses 479 during the query trials in the conditioning phase. Overall, we observed a slightly greater 480 sensitivity towards reward compared to punishment, suggesting that for some participants 481 seeking rewards was more relevant during the conditioning phase than avoiding punishment. 482 However, the overwhelming majority of participants did not exhibit a bias in any direction and 483 almost all participants were able to correctly assign the monetary values to the different face 484 exemplars until the end of the conditioning phase. Notably, in case of negative values 485 participants had to respond accurately to avoid monetary losses during the query trials. It is 486 possible that this task has rendered the loss-related faces similar to the reward-related faces in 487 terms of their motivational content. It has indeed been argued previously that the avoidance of 488 aversive outcomes can be rewarding (Kim, Shimojo \& O'Doherty, 2006), which would imply 489 that the loss-related faces in our study might have been perceived as motivationally positive. 490 This assumption is in conflict with several other studies, however, indicating that separate neural 491 structures underlie reward and avoidance learning (Yacubian et al., 2006; Palminteri et al., 2012; 492 Rothkirch et al., 2017), which suggests that the avoidance of punishment is qualitatively distinct 493 from receiving a reward.

494 For the assessment of possible asymmetries in the sensitivity between reward and punishment or 495 motivational similarities between seeking rewards and avoiding losses, it is important to take into 496 account that we compared response times in the bCFS phase between high and low values 497 separately for reward and punishment. Thus, any influences that might have affected the relation 498 between reward- and punishment-related faces could not have biased our results.

499

500 It is conceivable that an influence of learned values on response times might have been 501 dampened either due to habituation because of the repeated exposure to each face exemplar or 502 due to extinction during the post-conditioning phase. Furthermore, it has previously been 503 reported that responses to fear-conditioned faces rapidly decrease when these faces are visually 504 masked (Raio et al., 2012). Thus, effects of learned values can be obscured when responses are 505 aggregated across the post-conditioning phase. However, an analysis that distinguished between 506 the first and second half of the post-conditioning phase did not provide any indication of such 507 time-dependent effects in our study. Moreover, while Raio et al. (2012) conducted the 508 conditioning procedure with faces that were suppressed from awareness, which likely established 509 only unstable associations between the conditioned and unconditioned stimuli, the conditioning 510 procedure in our study was performed with fully visible face stimuli. Still, each face exemplar 511 was repeated 64 times across all phases in our study. Indeed, in comparison to previous studies 512 (Anderson et al., 2011; Rabovsky, Stein \& Abdel Rahman, 2016; Stein et al., 2017) our 513 conditioning phase comprised more repetitions of each face exemplar and a lower number of 514 different face exemplars. It has to be noted, however, that this ensured that participants 
515 successfully learned the associations between faces and values. Rabovsky, Stein \& Abdel

516 Rahman (2016), in contrast, report that in their study only $36 \%$ of newly learned associations

517 could be explicitly recalled after the learning phase, on average. In the other two studies

518 (Anderson et al., 2011; Stein et al., 2017), the learning procedure was repeated until participants

519 reached a criterion of at least $60 \%$ correct responses. Consequently, participants differed in the

520 frequency with which they were exposed to the face stimuli. Furthermore, they underwent the

521 post-conditioning task even though some of them might not have learned the correct associations

522 for a substantial amount of faces until the end of the learning phase. This suggests that fewer

523 repetitions during the learning phase likely come at the expense of a poorer learning

524 performance, which could have also contributed to the discrepant findings in previous studies.

525 According to Vansteenwegen et al. (2006), the affective content that faces gain through classical

526 conditioning is further largely resistant to extinction, at least at the behavioral level. In their first

527 experiment, response times in an affective priming task were still influenced by the learned

528 values after extinction, even though the experiment comprised 60 repetitions of each of the two

529 different conditioned face stimuli in total, which is comparable to the 64 repetitions of each face

530 exemplar in our study.

531

532 While we used faces with a neutral expression in our study, a potential approach to further

533 strengthen the association between the faces and the monetary values is to use faces with an

534 emotional expression. As suggested by the 'preparedness hypothesis', faces with different

535 emotional expressions might be differentially prepared to become associated with different

536 outcomes (Dimberg \& Öhman, 1996). In this context, pairing aversive outcomes with angry

537 faces, for instance, might be more effective than pairing them with neutral faces. The specific

538 interactions between different emotional expressions and monetary outcomes have not been

539 systematically studied yet, however, and such an approach my come at the expense of potential

540 ceiling effects (Lonsdorf et al., 2017). Finally, while we have used monetary outcomes to render

541 face stimuli behaviorally relevant, the use of other reinforcers, like liquid rewards in water-

542 deprived participants or bursts of white noise, are conceivable alternatives. We chose monetary

543 outcomes as they are easy to administer and can be equally employed as rewarding and aversive

544 stimuli. Furthermore, the processing of primary and secondary reinforcers, including monetary

545 values, shows large overlaps in the human brain (Izuma, Saito \& Sadato, 2008; Delgado, Jou \&

546 Phelps, 2011; Sescousse et al., 2013), which suggests that monetary values can evoke similar

547 positive or negative experiences in comparison to other types of reinforcers.

548

549

550 Conclusions

551

552 To conclude, we did not observe a privileged access to awareness for faces that were associated 553 with positive or negative monetary outcomes, although participants quickly learned these

554 associations. This tentatively suggests that learned values that are tied to a face's identity have 
555

556

557

558

559

560

561

562

563

564

565

566

567

568

569

570

571

572

573

574

575

576

577

578

579

580

581

582

583

584

585

586

587

588

589

590

591

592

593

594

595

596

597

only limited influence on the face's access to awareness, as such an influence possibly exceeds the scope of pre-conscious processing.

\section{References}

Amihai I, Deouell L, Bentin S. 2011. Conscious awareness is necessary for processing race and gender information from faces. Consciousness and Cognition 20:269-279. DOI: 10.1016/j.concog.2010.08.004.

Anderson E, Siegel EH, Bliss-Moreau E, Barrett LF. 2011. The visual impact of gossip. Science (New York, N.Y.) 332:1446-1448. DOI: 10.1126/science.1201574.

Austin AJ, Duka T. 2010. Mechanisms of attention for appetitive and aversive outcomes in Pavlovian conditioning. Behavioural Brain Research 213:19-26. DOI: 10.1016/j.bbr.2010.04.019.

Axelrod V, Bar M, Rees G. 2015. Exploring the unconscious using faces. Trends in Cognitive Sciences 19:35-45. DOI: 10.1016/j.tics.2014.11.003.

Barr DJ, Levy R, Scheepers C, Tily HJ. 2013. Random effects structure for confirmatory hypothesis testing: Keep it maximal. Journal of Memory and Language 68:255-278. DOI: 10.1016/j.jml.2012.11.001.

Bender R, Lange S. 2001. Adjusting for multiple testing--when and how? Journal of Clinical Epidemiology 54:343-349. DOI: 10.1016/s0895-4356(00)00314-0.

Bucker B, Theeuwes J. 2016. Appetitive and aversive outcome associations modulate exogenous cueing. Attention, Perception \& Psychophysics 78:2253-2265. DOI: 10.3758/s13414-016-1107-6.

Bucker B, Theeuwes J. 2017. Pavlovian reward learning underlies value driven attentional capture. Attention, Perception \& Psychophysics 79:415-428. DOI: 10.3758/s13414-0161241-1.

Bucker B, Theeuwes J. 2018. Stimulus-driven and goal-driven effects on Pavlovian associative reward learning. Visual Cognition 26:131-148. DOI: 10.1080/13506285.2017.1399948.

Delgado MR, Jou RL, Phelps EA. 2011. Neural systems underlying aversive conditioning in humans with primary and secondary reinforcers. Frontiers in Neuroscience 5:71. DOI: 10.3389/fnins.2011.00071.

Delgado MR, Labouliere CD, Phelps EA. 2006. Fear of losing money? Aversive conditioning with secondary reinforcers. Social Cognitive and Affective Neuroscience 1:250-259. DOI: 10.1093/scan/nsl025.

Dimberg U, Öhman A. 1996. Behold the wrath: Psychophysiological responses to facial stimuli. Motivation and Emotion 20:149-182. DOI: 10.1007/BF02253869.

Gayet S, Paffen CLE, Belopolsky AV, Theeuwes J, Van der Stigchel S. 2016. Visual input signaling threat gains preferential access to awareness in a breaking continuous flash suppression paradigm. Cognition 149:77-83. DOI: 10.1016/j.cognition.2016.01.009.

Gayet S, Stein T. 2017. Between-Subject Variability in the Breaking Continuous Flash Suppression Paradigm: Potential Causes, Consequences, and Solutions. Frontiers in Psychology 8:437. DOI: 10.3389/fpsyg.2017.00437. 
598

599

600

601

602

603

604

605

606

607

608

609

610

611

612

613

614

615

616

617

618

619

620

621

622

623

624

625

626

627

628

629

630

631

632

633

634

635

636

637

638

639

640

641

Getov S, Kanai R, Bahrami B, Rees G. 2015. Human brain structure predicts individual differences in preconscious evaluation of facial dominance and trustworthiness. Social Cognitive and Affective Neuroscience 10:690-699. DOI: 10.1093/scan/nsu103.

Gray KLH, Adams WJ, Hedger N, Newton KE, Garner M. 2013. Faces and awareness: lowlevel, not emotional factors determine perceptual dominance. Emotion (Washington, D.C.) 13:537-544. DOI: 10.1037/a0031403.

Han S, Blake R, Alais D. 2018. Slow and steady, not fast and furious: Slow temporal modulation strengthens continuous flash suppression. Consciousness and Cognition 58:10-19. DOI: 10.1016/j.concog.2017.12.007.

Hedger N, Gray KLH, Garner M, Adams WJ. 2016. Are visual threats prioritized without awareness? A critical review and meta-analysis involving 3 behavioral paradigms and 2696 observers. Psychological Bulletin 142:934-968. DOI: 10.1037/bul0000054.

Izuma K, Saito DN, Sadato N. 2008. Processing of social and monetary rewards in the human striatum. Neuron 58:284-294. DOI: 10.1016/j.neuron.2008.03.020.

Kim H, Shimojo S, O'Doherty JP. 2006. Is Avoiding an Aversive Outcome Rewarding? Neural Substrates of Avoidance Learning in the Human Brain. PLOS Biology 4:e233. DOI: 10.1371/journal.pbio.0040233.

Leganes-Fonteneau M, Scott R, Duka T. 2018. Attentional responses to stimuli associated with a reward can occur in the absence of knowledge of their predictive values. Behavioural Brain Research 341:26-36. DOI: 10.1016/j.bbr.2017.12.015.

Lehner R, Balsters JH, Herger A, Hare TA, Wenderoth N. 2016. Monetary, Food, and Social Rewards Induce Similar Pavlovian-to-Instrumental Transfer Effects. Frontiers in Behavioral Neuroscience 10:247. DOI: 10.3389/fnbeh.2016.00247.

Lo S, Andrews S. 2015. To transform or not to transform: using generalized linear mixed models to analyse reaction time data. Frontiers in Psychology 6. DOI: 10.3389/fpsyg.2015.01171.

Lonsdorf TB, Menz MM, Andreatta M, Fullana MA, Golkar A, Haaker J, Heitland I, Hermann A, Kuhn M, Kruse O, Meir Drexler S, Meulders A, Nees F, Pittig A, Richter J, Römer S, Shiban Y, Schmitz A, Straube B, Vervliet B, Wendt J, Baas JMP, Merz CJ. 2017. Don't fear "fear conditioning": Methodological considerations for the design and analysis of studies on human fear acquisition, extinction, and return of fear. Neuroscience and Biobehavioral Reviews 77:247-285. DOI: 10.1016/j.neubiorev.2017.02.026.

Madipakkam AR, Rothkirch M. 2019. The Unconscious Processing of Social Information. In: Transitions Between Consciousness and Unconsciousness. DOI: 10.4324/9780429469688-4.

Madipakkam AR, Rothkirch M, Dziobek I, Sterzer P. 2019. Access to awareness of direct gaze is related to autistic traits. Psychological Medicine 49:980-986. DOI: 10.1017/S0033291718001630.

Madipakkam AR, Rothkirch M, Wilbertz G, Sterzer P. 2016. Probing the influence of unconscious fear-conditioned visual stimuli on eye movements. Consciousness and Cognition 46:60-70. DOI: 10.1016/j.concog.2016.09.016.

Moors P, Gayet S, Hedger N, Stein T, Sterzer P, van Ee R, Wagemans J, Hesselmann G. 2019. Three Criteria for Evaluating High-Level Processing in Continuous Flash Suppression. Trends in Cognitive Sciences 23:267-269. DOI: 10.1016/j.tics.2019.01.008.

Peer) reviewing PDF | (2020:06:49896:3:0:NEW 7 Jan 2021) 
642 Moradi F, Koch C, Shimojo S. 2005. Face adaptation depends on seeing the face. Neuron

643

644

645

646

647

648

649

650

651

652

653

654

655

656

657

658

659

660

661

662

663

664

665

666

667

668

669

670

671

672

673

674

675

676

677

678

679

680

681

682

683

684

685 45:169-175. DOI: 10.1016/j.neuron.2004.12.018.

Palminteri S, Justo D, Jauffret C, Pavlicek B, Dauta A, Delmaire C, Czernecki V, Karachi C, Capelle L, Durr A, Pessiglione M. 2012. Critical Roles for Anterior Insula and Dorsal Striatum in Punishment-Based Avoidance Learning. Neuron 76:998-1009. DOI: 10.1016/j.neuron.2012.10.017.

Pietrock C, Ebrahimi C, Katthagen TM, Koch SP, Heinz A, Rothkirch M, Schlagenhauf F. 2019. Pupil dilation as an implicit measure of appetitive Pavlovian learning. Psychophysiology 56:e13463. DOI: 10.1111/psyp.13463.

Rabovsky M, Stein T, Abdel Rahman R. 2016. Access to Awareness for Faces during Continuous Flash Suppression Is Not Modulated by Affective Knowledge. PloS One 11:e0150931. DOI: 10.1371/journal.pone.0150931.

Raio CM, Carmel D, Carrasco M, Phelps EA. 2012. Nonconscious fear is quickly acquired but swiftly forgotten. Current biology: CB 22:R477-479. DOI: 10.1016/j.cub.2012.04.023.

Raymond JE, O'Brien JL. 2009. Selective visual attention and motivation: the consequences of value learning in an attentional blink task. Psychological Science 20:981-988. DOI: 10.1111/j.1467-9280.2009.02391.x.

Rehbein MA, Pastor MC, Moltó J, Poy R, López-Penadés R, Junghöfer M. 2018. Identity and expression processing during classical conditioning with faces. Psychophysiology 55:e13203. DOI: 10.1111/psyp.13203.

Rothkirch M, Ostendorf F, Sax A-L, Sterzer P. 2013. The influence of motivational salience on saccade latencies. Experimental Brain Research 224:35-47. DOI: 10.1007/s00221-0123284-4.

Rothkirch M, Schmack K, Schlagenhauf F, Sterzer P. 2012. Implicit motivational value and salience are processed in distinct areas of orbitofrontal cortex. Neurolmage 62:17171725. DOI: 10.1016/j.neuroimage.2012.06.016.

Rothkirch M, Tonn J, Köhler S, Sterzer P. 2017. Neural mechanisms of reinforcement learning in unmedicated patients with major depressive disorder. Brain: A Journal of Neurology 140:1147-1157. DOI: 10.1093/brain/awx025.

Rousselet GA, Wilcox RR. 2019. Reaction times and other skewed distributions: problems with the mean and the median. PsyArXiv. DOI: 10.31234/osf.io/3y54r.

Rutherford HJV, O'Brien JL, Raymond JE. 2010. Value associations of irrelevant stimuli modify rapid visual orienting. Psychonomic Bulletin \& Review 17:536-542. DOI: 10.3758/PBR.17.4.536.

Schmack K, Burk J, Haynes J-D, Sterzer P. 2016. Predicting Subjective Affective Salience from Cortical Responses to Invisible Object Stimuli. Cerebral Cortex 26:3453-3460. DOI: 10.1093/cercor/bhv174.

Schönbrodt FD, Wagenmakers E-J, Zehetleitner M, Perugini M. 2017. Sequential hypothesis testing with Bayes factors: Efficiently testing mean differences. Psychological Methods 22:322-339. DOI: 10.1037/met0000061.

Sescousse G, Caldú X, Segura B, Dreher J-C. 2013. Processing of primary and secondary rewards: a quantitative meta-analysis and review of human functional neuroimaging studies. Neuroscience and Biobehavioral Reviews 37:681-696. DOI: 10.1016/j.neubiorev.2013.02.002. 
686

687

688

689

690

691

692

693

694

695

696

697

698

699

700

701

702

703

704

705

706

707

708

709

710

711

712

713

714

715

716

717

718

719

720

721

722

723

Stein T, Awad D, Gayet S, Peelen MV. 2018. Unconscious processing of facial dominance: The role of low-level factors in access to awareness. Journal of Experimental Psychology. General 147:e1-e13. DOI: 10.1037/xge0000521.

Stein T, Grubb C, Bertrand M, Suh SM, Verosky SC. 2017. No impact of affective person knowledge on visual awareness: Evidence from binocular rivalry and continuous flash suppression. Emotion (Washington, D.C.) 17:1199-1207. DOI: 10.1037/emo0000305.

Stein T, Sterzer P. 2012. Not just another face in the crowd: detecting emotional schematic faces during continuous flash suppression. Emotion (Washington, D.C.) 12:988-996. DOI: 10.1037/a0026944.

Stewart LH, Ajina S, Getov S, Bahrami B, Todorov A, Rees G. 2012. Unconscious evaluation of faces on social dimensions. Journal of Experimental Psychology. General 141:715-727. DOI: $10.1037 / \mathrm{a} 0027950$.

Tottenham N, Tanaka JW, Leon AC, McCarry T, Nurse M, Hare TA, Marcus DJ, Westerlund A, Casey BJ, Nelson C. 2009. The NimStim set of facial expressions: judgments from untrained research participants. Psychiatry Research 168:242-249. DOI: 10.1016/j.psychres.2008.05.006.

Trilla Gros I, Panasiti MS, Chakrabarti B. 2015. The plasticity of the mirror system: how reward learning modulates cortical motor simulation of others. Neuropsychologia 70:255-262. DOI: 10.1016/j.neuropsychologia.2015.02.033.

Vansteenwegen D, Francken G, Vervliet B, De Clercq A, Eelen P. 2006. Resistance to extinction in evaluative conditioning. Journal of Experimental Psychology. Animal Behavior Processes 32:71-79. DOI: 10.1037/0097-7403.32.1.71.

Vieira JB, Wen S, Oliver LD, Mitchell DGV. 2017. Enhanced conscious processing and blindsight-like detection of fear-conditioned stimuli under continuous flash suppression. Experimental Brain Research 235:3333-3344. DOI: 10.1007/s00221-017-5064-7.

Whalen PJ, Kagan J, Cook RG, Davis FC, Kim H, Polis S, McLaren DG, Somerville LH, McLean AA, Maxwell JS, Johnstone T. 2004. Human amygdala responsivity to masked fearful eye whites. Science (New York, N.Y.) 306:2061. DOI: 10.1126/science.1103617.

Yacubian J, Gläscher J, Schroeder K, Sommer T, Braus DF, Büchel C. 2006. Dissociable Systems for Gain- and Loss-Related Value Predictions and Errors of Prediction in the Human Brain. Journal of Neuroscience 26:9530-9537. DOI: 10.1523/JNEUROSCI.291506.2006.

Yang Y-H, Yeh S-L. 2018. Can emotional content be extracted under interocular suppression? PloS One 13:e0206799. DOI: 10.1371/journal.pone.0206799.

Yang E, Zald DH, Blake R. 2007. Fearful expressions gain preferential access to awareness during continuous flash suppression. Emotion (Washington, D.C.) 7:882-886. DOI: 10.1037/1528-3542.7.4.882.

Peer) reviewing PDF | (2020:06:49896:3:0:NEW 7 Jan 2021) 


\section{Figure 1}

Schematic depiction of the experimental procedure.

(A) In the pre- and post-conditioning phase, high-contrast dynamic mask stimuli were presented to one eye at a frequency of $10 \mathrm{~Hz}$. A face stimulus was simultaneously presented to the other eye. The contrast of the face stimulus linearly increased during the initial $2 \mathrm{~s}$ and remained at full contrast until the end of the trial. Participants' task was to indicate the location of the face. A trial ended either after a manual response or at the latest after $15 \mathrm{~s}$. (B) In the conditioning phase, different face stimuli were presented together with their associated monetary outcome (upper part in panel B). Participants' task was to passively view and memorize these associations. This phase comprised four blocks. At the end of each block, query trials where performed (lower part in panel B), where each face stimulus was presented with four different response options. Here, participants had to select the value that was previously associated with the face. Due to copyright reasons, the original face images used in the experiment are not shown in the figure but replaced by a schematic drawing. 
A

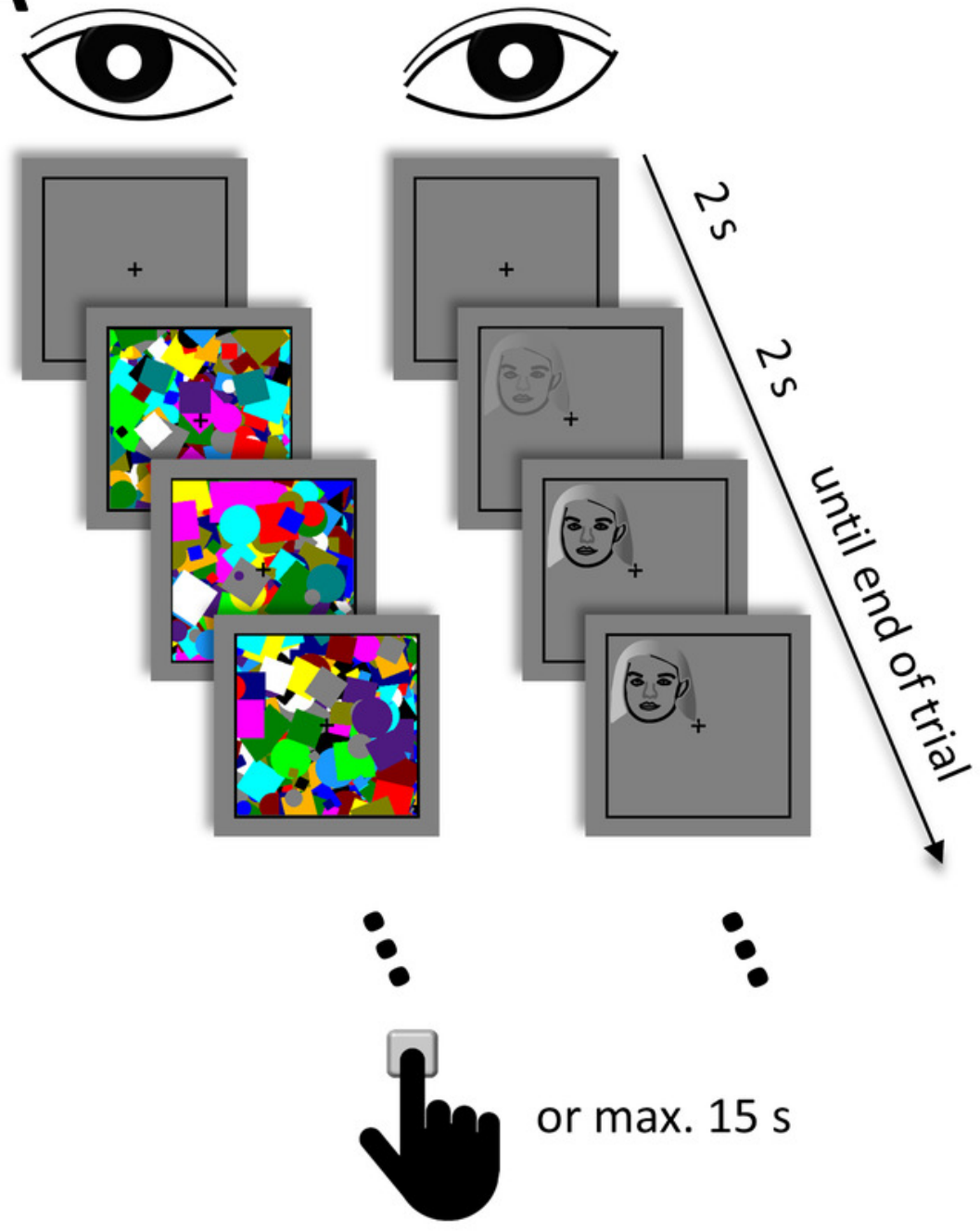

B

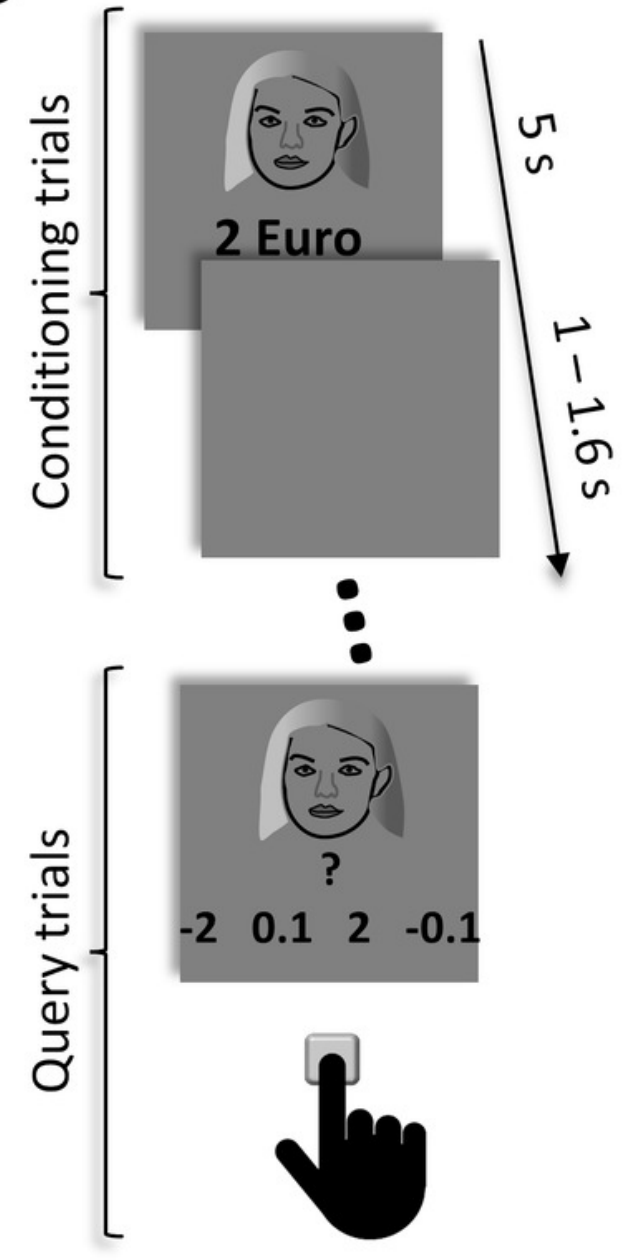


Figure 2

Choice accuracy in the query trials of the conditioning phase.

(A) Accuracy for each block of the conditioning phase. (B) Accuracy for each value condition. Each dot represents one participant.
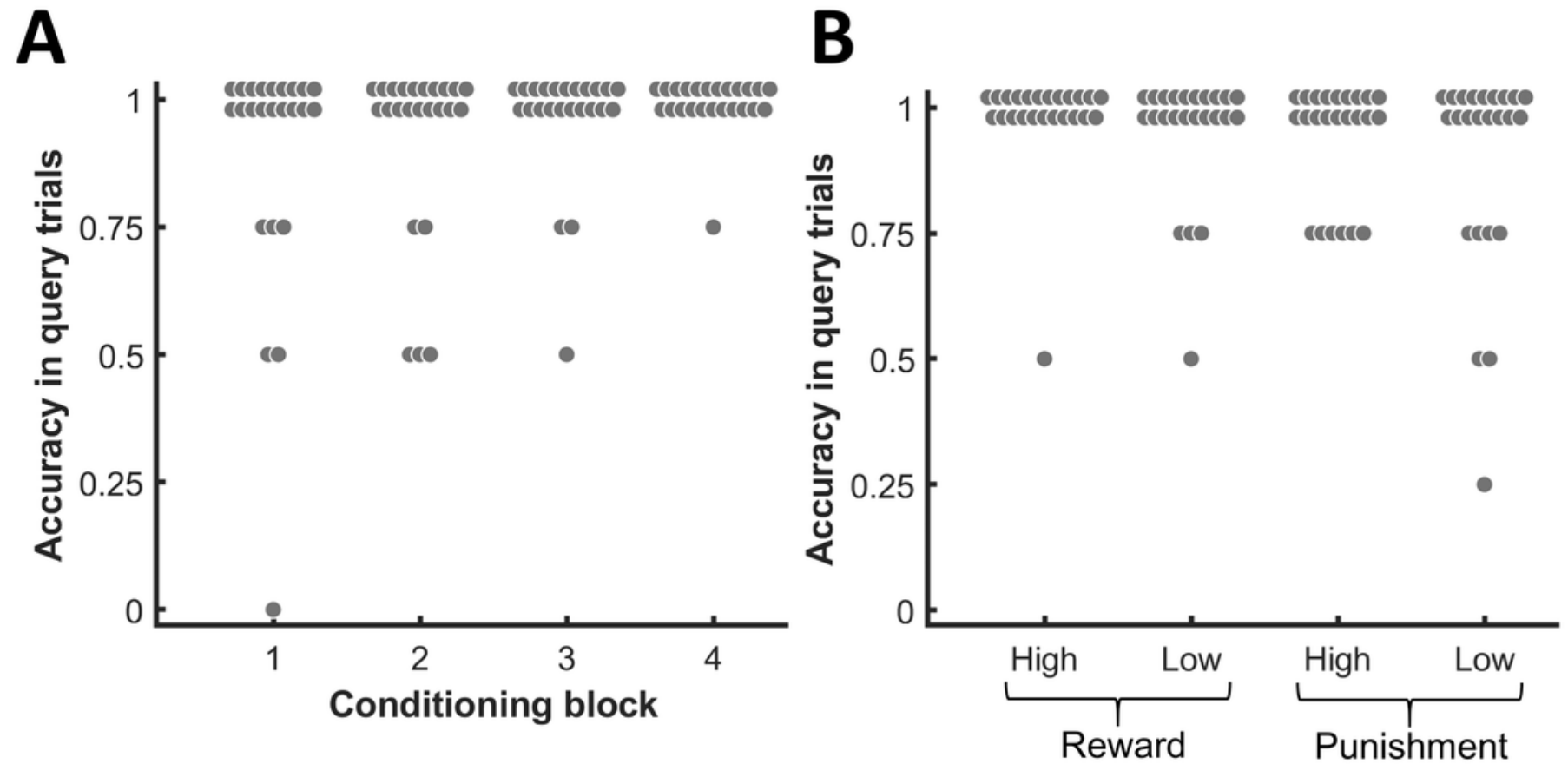


\section{Figure 3}

Change in response times (RT) for (A) the reward-related and (B) the punishmentrelated stimuli.

Negative values indicate an RT decrease in the post-conditioning phase compared to the preconditioning phase. The position of each data point is defined by the RT change for the high value condition on the $x$-axis and the RT change for the low value condition on the $y$-axis. Participants who showed a stronger RT reduction for the high in comparison to the low value condition (i.e. RT differences consistent with our a priori hypotheses) are located in the greyshaded area. The magenta-coloured dashed line indicates the mean RT change for the high value condition across participants. The turquoise dashed line indicates the mean RT change for the low value condition across participants.
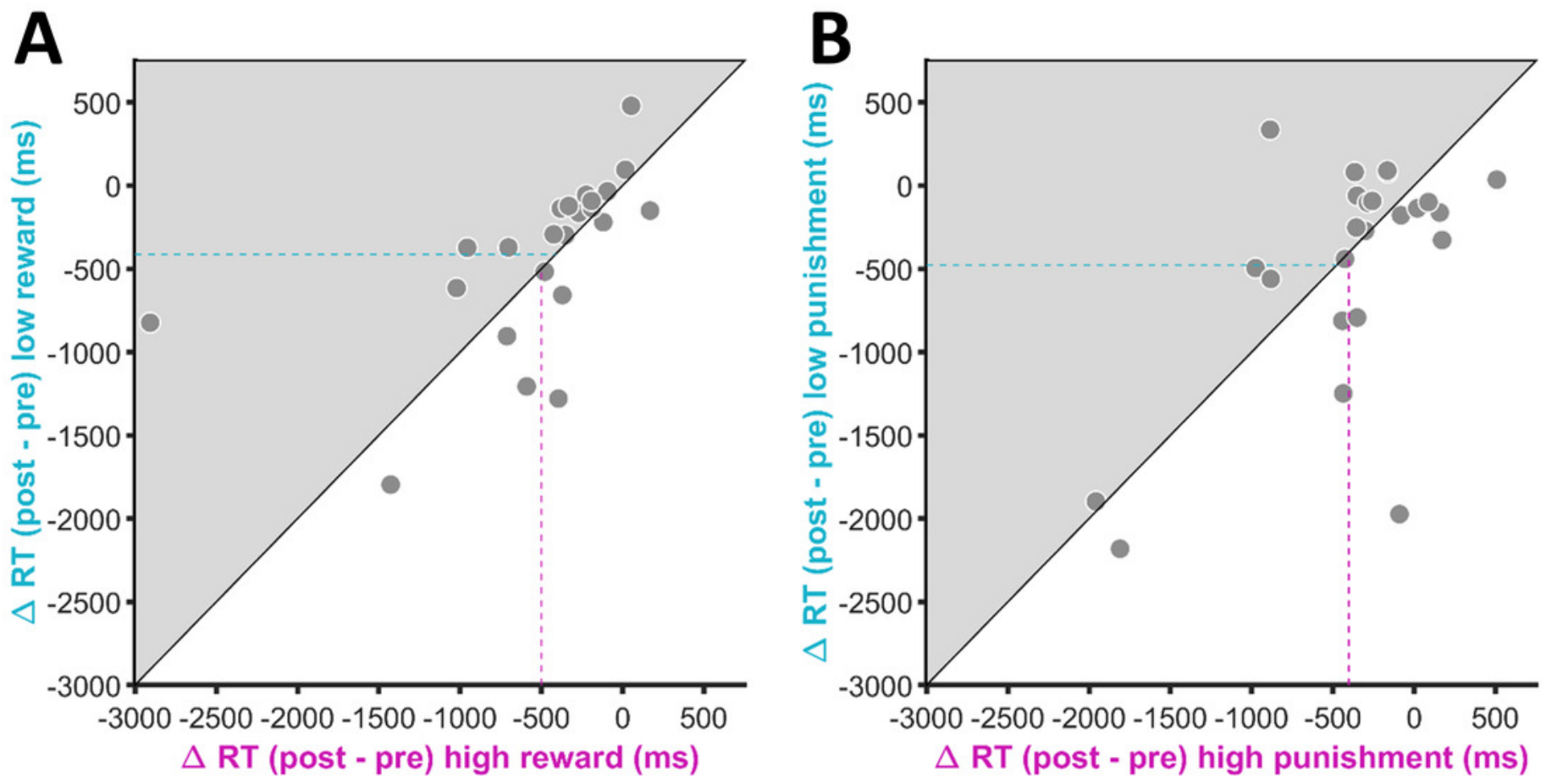
Figure 4

Change in response times for each decile of the whole response distributions for the $(A)$ reward and (B) punishment conditions.

The numbers at the top of each graph indicate the $p$-values and the Bayes Factors for the comparison between the two conditions for each respective decile. The error bars display standard errors of the mean.
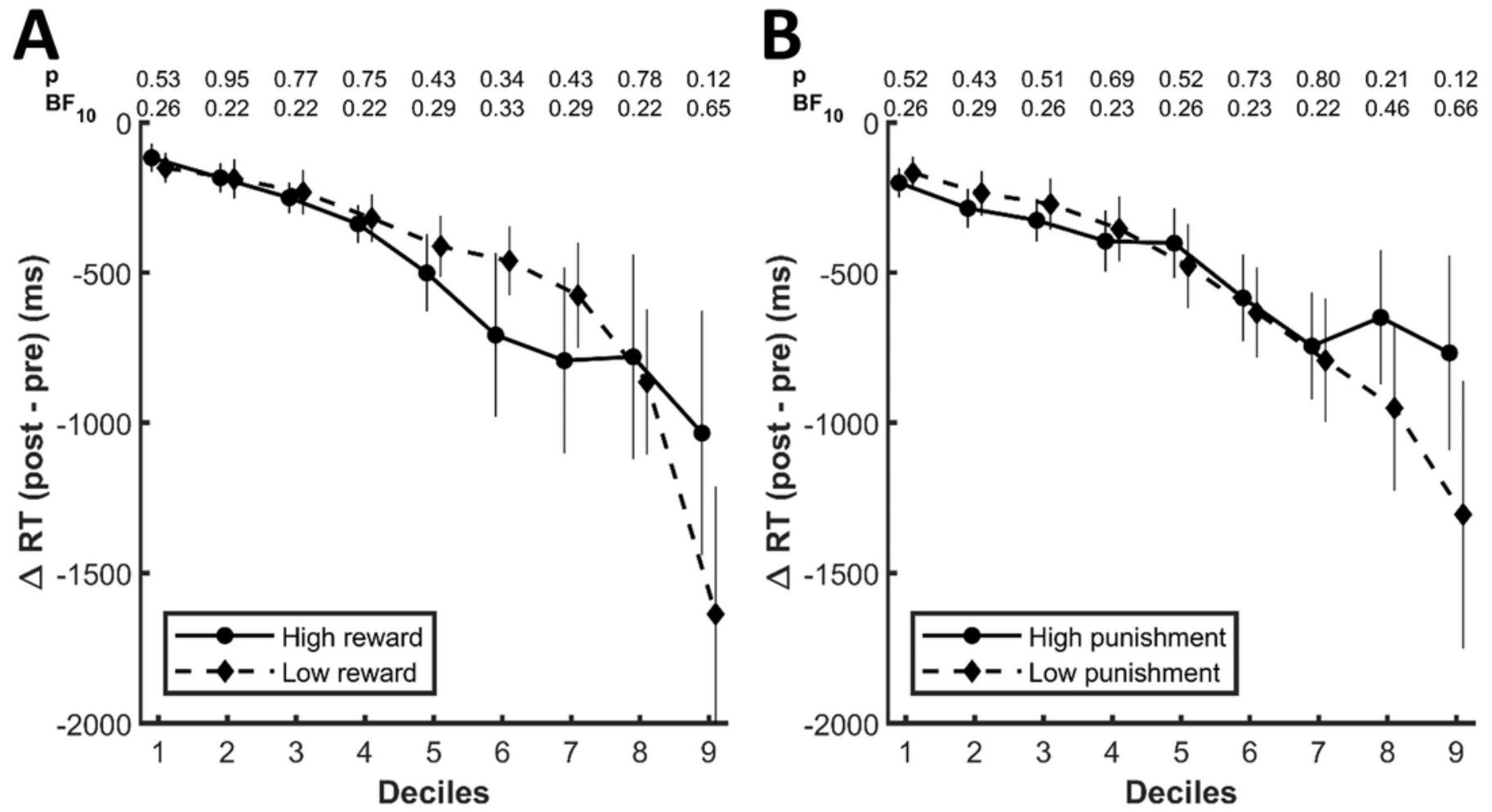